\title{
Cognitive Improved LEACH (CogILEACH) Protocol for Wireless Sensor Network
}

\author{
${ }^{1} \mathrm{Md}$ Sipon Miah, ${ }^{2}$ Dilruba Sharmin and ${ }^{3}$ Md Mahbubur Rahman \\ 1,3 Department of Information and Communication Engineering, Islamic University, Kushtia, Bangladesh; \\ ${ }^{2}$ Departmne of Information and Communication Engineering, College of Rangpur Engineering, Rajshahi \\ University, Rajshahi, Bangladesh; \\ sipon@ice.iu.ac.bd; dilrubasharminice@gmail.com; mrahman@ice.iu.ac.bd
}

\begin{abstract}
In this paper, we proposed the Cognitive Improved Low Energy Adaptive Clustering Hierarchy (CogILEACH) protocol that is the spectrum aware extension of the Low Energy Adaptive Clustering Hierarchy (LEACH) protocol. In CogILEACH, which selects a cluster head $(\mathrm{CH})$ based on its ratio between the current residual energy and an initial energy, and multiplies by the root square of its number of neighbor nodes. The simulation results show that CogILEACH protocol improves the lifetime of the network compared to both the LEACH protocol and ILEACH protocol under two-level homogeneity.
\end{abstract}

Keywords: LEACH protocol, ILEACH protocol, Cluster head, Cognitive radio, Wireless sensor networks

\section{Introduction}

The wireless sensor network (WSN) operates in the public unlicensed spectrum band, which has become increasingly crowd because of the emergence of the vast wireless communication technologies. In order to solve the above problems in WSN, the cognitive radio sensor networks (CRSN) is proposed [1-3].

CRSN Network has recently attracted a large amount of attention due to its advantage of dynamic spectrum access which provides reliability in terms of communication and enhances energy conservation potential in sensor network. Each node in a CSRN is typically equipped with a sensors having cognitive capability for communication purpose. Preserving the consumed energy of each node is an important goal that must be considered when developing any protocol for cognitive radio sensor networks [4-5].

Cognitive Radio (CR) enables opportunistic access to unused licensed bands. CR allows secondary users (SUs) to utilize the free portions of licensed spectrum while ensuring no interference to primary users (PUs) transmissions [6].

A CR is a radio that can change its transmitter parameters based on interaction with the environment it operates. It is able to monitor sense and detect the conditions of their operating environment and reconfigure their own characteristics dynamically and to best match those conditions. Also, it is a form of wireless communication in which a transceiver can intelligently detect which communication channels are in use and instantly move into vacant channels while avoiding occupied ones in order to optimize the use of available Radio-Frequency (RF) spectrum while minimizing interference to other users [7][8]. 
Md Sipon Miah, Dilruba Sharmin and Md Mahbubur Rahman; Cognitive Improved LEACH (CogILEACH) Protocol for Wireless Sensor Network, Transactions on Networks and Communications, Volume 4 No. 6, December (2016);

pp: 1-9

In a wireless communication system the electromagnetic spectrums becoming crowded day by day due to the increment of wireless applications. In which the cognitive radio plays a very important role for spectrum sensing. In which it is observed that the available spectrum is not utilized properly at that time and particular geographic location. To overcome this problem the cognitive radio technology is proposed to sense unused bands. CR is to provide effective communication and utilization of spectrum bands for users. CR having the ability to determine the presence of the licensed user, and also detects which portion of spectrum is available, in other words, to detect the white spaces that means empty bands, which is known as spectrum sensing [9].

Clustering in a CRSN is similar to clustering in an ordinary WSN. Each cluster consists of one cluster head and a number of cluster members. Each member node is attached to one of the $\mathrm{CH}$ node according to application-specific objectives (e.g. minimum distance $\mathrm{CH}$ to reduce transmission power) resulting in a cluster-shaped network. Member nodes send their data to their corresponding $\mathrm{CH}$ nodes instead of sending the data directly to the Base Station (BS) (i.e. reduce communication energy). Subsequently, $\mathrm{CH}$ nodes send these packets to the BS either in single-hop or multi-hop [10].

In this paper, we introduce CogILEACH, which is a probabilistic clustering algorithm that selects a $\mathrm{CH}$ based on its ratio between the current residual energy and an initial energy, and multiplies by the root square of its number of neighbor nodes. CogILEACH supports different network and PU models, and accounts for network scalability. CogILEACH is a spectrum-aware extension of the legacy LEACH protocol Simulation results show that CogILEACH improves the lifetime of the network compared to the LEACH protocol and ILEACH protocol under two-level homogeneity.

The remainder of this paper is as follows: we discuss the existing LEACH protocol in Section 2 and Section 3 we analytically introduce the proposed CogILEACH protocol structure. In Section 4, we describe our model parameters and assumptions, and evaluate the performance through Simulations. In Section 5, we discuss results analysis and discussion. Finally, Section 5 concludes the paper.

\section{The Existing LEACH Protocol}

LEACH is one of the most popular cluster-based routing protocols in wireless sensor networks that minimize global energy usage by distributing the load to all nodes at different points in time. The operation of LEACH consists of cycles, where each cycle consists of $\frac{N}{k}$ rounds. In LEACH each node decides its state ( $\mathrm{CH}$ or member node) autonomously through the following probability:

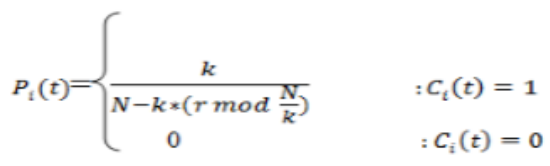

where $N$ denotes the total number of nodes, $k$ denotes the desired number of $\mathrm{CHs}$ (on average) per round, $r$ denotes the current round number, and $C_{i}(t)$ is an indicator function determining whether or not node $i$ has been a $\mathrm{CH}$ for the most recent $r \bmod \left(\frac{N}{k}\right)$ rounds. This formula of $P_{i}(t)$ maintains that each node is selected as a $\mathrm{CH}$ once per cycle of operation and thus balancing the load between nodes and extending time of the first node death. LEACH shows significant improvements to Minimum Transmission Energy (MTE) routing, but LEACH protocol is designed for a WSN operating on a fixed channel and thus not URL: $\underline{\text { http://dx.doi.org/10.14738/tnc.46.2301 }}$ 
applicable for CRSN. However, the main problem of the LEACH protocol lies in the random selection of $\mathrm{CHs}$. There exists a probability that the $\mathrm{CH}$ formation is unbalanced and may remain in one part of the network, and then it makes some part of the network be unreachable. Also one-hop inter-cluster and intra-cluster used in LEACH is not applicable for large part of the network [11-12].

LEACH-B (Balanced) which proposes an enhanced version of LEACH by finding the number of CHs which are near optimal. In LEACH-B, there is a second stage for selecting $\mathrm{CH}$ s through considering the residual energy of candidate nodes to become $\mathrm{CH}$. LEACH-B works similar to LEACH by selecting a random number between 0 and 1 and calculating the threshold value. The simulation results showed that there is performance improvement by using LEACH-B [13]. The disadvantage of this protocol is that extra overhead for electing $\mathrm{CHs}$.

An improved LEACH scheme was proposed with a name of LEACH-C (Centralized). Recall that in LEACH all nodes select $\mathrm{CH}$ s by themselves and the result will be a number of $\mathrm{CHs}$, some normal nodes, and formed clusters. In LEACH-C, authors made an improvement over LEACH protocol such that during the rounds of the $\mathrm{CH}$ selection stage the BS should know the remaining energy of all the nodes and there location. Accordingly, the BS selects the most suitable nodes to be $\mathrm{CHs}$, and divides the rest of the nodes between $\mathrm{CHs}$ to form the clusters. Simulation results of LEACH-C shows an enhancement based on the first dead node compared to LEACH and the traditional routing protocols (MTE and static clustering).since most of the energy is consumed in the BS. Extra overhead on the BS. LAECH-C does not fit for large area networks [14].

Further, the clustering protocol known as LEACH-E was proposed. In this protocol, the $\mathrm{CHs}$ are elected according to the energy left in each node. The drawback of LEACH-E is that it requires the assistance of a routing protocol which should inform the total energy of network to each node [15].

Distributed energy efficient clustering (DEEC) is a dedicated design for energy heterogeneous scenarios where nodes are initialized at various energy levels. Decentralized energy efficient clustering propagation (DEEP) prevents cluster heads from being too close to each other, but ignores a cluster head's energy qualification [16].

PEGASIS which makes a communication chain using a traveling sales person heuristic. Each node only communicates with two close neighbors along the communication chain only a single designated node gathers data from other nodes and transmits the aggregated data to the sink node [17].

$\mathrm{CH}$ election is based on nodes residual energy as a primary clustering parameter, and node proximity to its neighbors as a secondary parameter. Hybrid Energy Efficient. Distributed (HEED) protocol shows significant improvements to LEACH in terms of lifetime and throughput, but it is designed for fixed channel settings and thus not applicable for CRSN [18].

SEP, which was proposed in [19], is a heterogeneous aware protocol based on weighted election probabilities of each node to become a $\mathrm{CH}$ according to their respective energy. This approach ensures that the $\mathrm{CH}$ election is randomly selected and distributed based on the fraction of energy of each node assuring a uniform use of the nodes energy. In SEP, however two level hierarchies are considered.

The low-energy adaptive uneven clustering hierarchy (LEAUCH) is proposed in [20] for CRSN. It can not only consider the advantage of channel resources brought by cognitive function in CRSN but also exploit 
Md Sipon Miah, Dilruba Sharmin and Md Mahbubur Rahman; Cognitive Improved LEACH (CogILEACH) Protocol for Wireless Sensor Network, Transactions on Networks and Communications, Volume 4 No. 6, December (2016);

pp: 1-9

the uneven clustering method based on the channel resources. The disadvantage is that cannot applicable for more complicated network topology.

A spectrum-aware clustering algorithm for a cognitive radio ad-hoc network (CRAN) is introduced. The solution is based on finding the optimal silique graph at each node subject to set of constraints. Clusters are formed with the aim of maximizing the summation of the number of common idle channels with the number of nodes within the cluster, or maximizing the multiplication of the same two parameters, or maximizing the number of nodes within the cluster given a threshold on the number of common idle channels. This solution is deterministic and it provides a stable clustering scheme, but it is NP-Hard and violates WSN main design constraints: energy and computation [21].

A spectrum-aware clustering scheme for CRSN is proposed by introducing Group Wise Constraint (GWC) to the legacy K-means clustering algorithm. GWC states that nodes within each cluster must have common idle channels and be in physical proximity relative to each other's. The protocol initially sets each node as a $\mathrm{CH}$ and then merges $\mathrm{CHs}$ within each iteration until the number of $\mathrm{CHs}$ reaches an optimal number that was obtained apriority by theoretical analysis. Information about physical proximity and common channels is sent from nodes to their corresponding $\mathrm{CHs}$. Then, a $\mathrm{CH}$ node can determine which cluster should be merged with it. Such information requires intensive exchange of messages between nodes and $\mathrm{CHs}$ and thus not energy efficient [22].

\section{The Proposed CogILEACH Protocol Structure}

The structure of CogILEACH Protocol is discussed in the following section.

\subsection{System Model}

In this section, we describe the model of Cognitive ILEACH protocol.

\subsubsection{Network Model}

The network consists of $N$ CRSN nodes that act as a secondary system existing with $P$ PUs operating in the same band over a set $C$ of orthogonal frequency channels. We define Interference Protection Range (IPR) for each Active PU as an area circulating each PU, no other radio inside the IPR of PUi could be tuned to the channel used by PUi to avoid interference. Thus any CRSN within the IPR of an active PUi will sense the channel used by this PU as busy channel.

\subsubsection{Energy Model}

For this analysis, we use the first order radio model. The transmission cost $E_{T X}(l, d)$ and reception cost $E_{R X}(l)$ of $I$ bits message between two nodes with their distance of $d$ are considered. The energy consumption of a transmit side is given by:

$$
E_{T X}(l, d)=\left\{\begin{array}{l}
l E_{\text {elec }}+l \varepsilon_{f s} d^{2}, \text { if } d \leq d_{0} \\
l E_{\text {elec }}+l \varepsilon_{f s} d^{2}, \text { if } d>d_{0}
\end{array}\right.
$$

The receiver energy consumption of receiving / data is: 


$$
E_{T X}(l, d)=l E_{\text {elec }}
$$

where the parameter $E_{\text {elec }}$ is the per bit energy dissipations for transmission and reception. We also use the free-space and multipath fading model according to the distance between the transmitter and receiver. One suitable choice for a threshold transmission distance $d_{0}$ may be: $d_{0}=\sqrt{\varepsilon_{f s} / \varepsilon_{a m p}}$. Where $\varepsilon_{f s}$ is the power loss of free space, and $\varepsilon_{a m p}$ is the power loss of multipath fading model models. If $d \leq$ $d_{0}$, the free-space model will be employed; otherwise, the multipath fading model will be employed as shown in Figure 1.1.

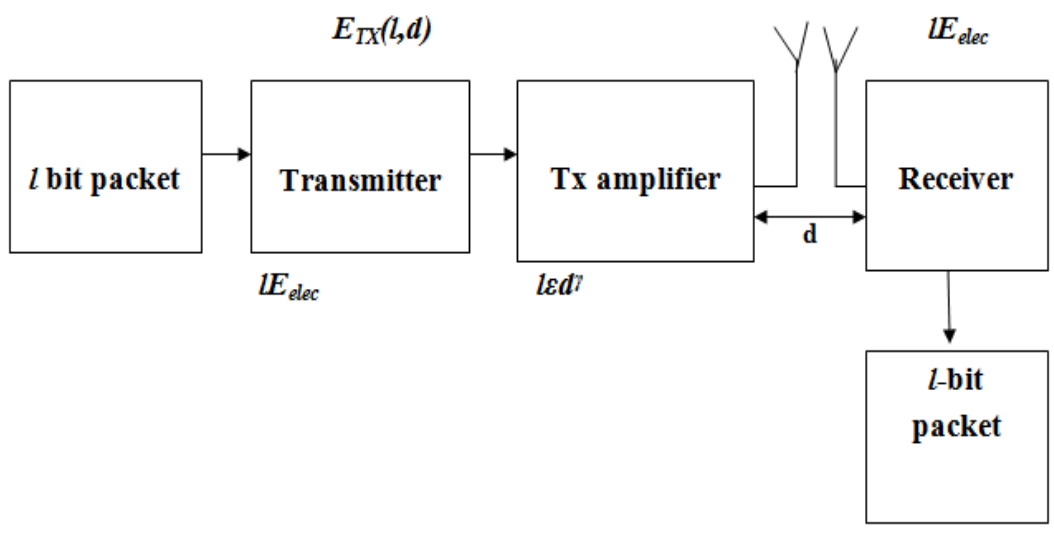

Figure 1: The radio signal propagation model.

\subsection{CogILEACH Protocol}

We propose the CogILEACH protocol which is based on the initial energy, the current residual energy and the number of neighbors of the nodes. CogILEACH protocol assigned a weight probability of a normal node, an intermediate node, and an advanced node. In the proposed CogILEACH scheme, the initial energy for normal nodes is $E_{n r m}=E_{0}$.For an intermediate and advanced nodes, $E_{i t m}=(1+\mu) E_{0}$ and $E_{a d v}=$ $(1+\alpha) E_{0}$ respectively. Thus, the total initial energy of the system $E_{\text {Total }}$ is given by:

$$
\begin{gathered}
E_{\text {Total }}=N f E_{0}(1+\alpha)+N g E_{0}(1+\mu)+N E_{0}(1-f-g) \\
=(1+\alpha f+\mu g) N E_{0} \quad \text { When } \mu=\alpha / 2
\end{gathered}
$$

where $f$ is the proportion of advanced nodes to the total number of nodes $N$ with energy more than the remaining of the nodes, and $g$ is the proportion of intermediate nodes. The weighted probability for normal node can be defined by

$$
P_{n r m}=\frac{P}{(1+f \alpha+g \mu)}
$$

We define the threshold for normal nodes, $T\left(S_{n r m}\right)$ as follows.

$$
\begin{gathered}
T\left(S_{n r m}\right)=\left\{\begin{array}{cc}
\frac{P_{\text {nrm }}}{1-P_{\text {nrm }}\left(\text { r mod } \frac{1}{P_{\text {nrm }}}\right)} E_{\text {ratio }}^{\text {nrm }}, & : C_{i}(t)=1 \\
0, & : C_{i}(t)=0
\end{array}\right. \\
E_{\text {ratio }}^{\text {nrm }}=\frac{E_{c}}{E_{0}} \sqrt{N_{i}}
\end{gathered}
$$




\section{pp: $1-9$}

where, $T\left(S_{n r m}\right)$ is the threshold applied to a population of $N(1-f-g)$ for normal nodes, $E_{0}$ is the initial energy, $E_{c}$ is the current residual energy, and $N_{i}$ is the number of neighbors of the $i$-th node. The average number of $\mathrm{CHs}$ for normal nodes per round per epoch is given by

$$
N(1-f-g) P_{n r m}
$$

The weight probability for intermediate node can be defined by

$$
P_{i t m}=\frac{P}{(1+f \alpha+g \mu)}(1+\mu)
$$

We define the threshold for intermediate nodes, $T\left(S_{i t m}\right)$ as follows

$$
\begin{gathered}
T\left(S_{\text {itm }}\right)= \begin{cases}\frac{P_{\text {itm }}}{1-P_{\text {itm }}\left(\text { rmod } \frac{1}{P_{\text {itm }}}\right)} E_{\text {ratio }}^{i t m}, & : C_{i}(t)=1 \\
0, & : C_{i}(t)=0\end{cases} \\
E_{\text {ratio }}^{\text {itm }}=\frac{E_{c}}{E_{0}(1+\mu)} \sqrt{N_{i}}
\end{gathered}
$$

where, $T\left(S_{i t m}\right)$ is the threshold applied to a population of $N g(1+\mu)$ for intermediate nodes. The average number of $\mathrm{CHs}$ for intermediate nodes per round per epoch is

$$
\mathrm{Ng} P_{\text {itm }}
$$

Here, $g$ is the proportion of intermediate nodes and $P_{i t m}$ is the weight probability of intermediate node. The weight probability for advanced node can be defined by

$$
P_{a d v}=\frac{P}{(1+f \alpha+g \mu)}(1+\alpha)
$$

We define the threshold for advanced nodes, $T\left(S_{a d v}\right)$ as follows.

$$
\begin{gathered}
T\left(S_{a d v}\right)=\left\{\begin{array}{cc}
\frac{P_{a d v}}{1-P_{a d v}\left(r \bmod \frac{1}{P_{a d v}}\right)} E_{\text {ratio }}^{a d v}, & : C_{i}(t)=1 \\
0, & : C_{i}(t)=0
\end{array}\right. \\
E_{\text {ratio }}^{a d v}=\frac{E_{c}}{(1+\alpha) E_{0}} \sqrt{N_{i}}
\end{gathered}
$$

where, $T\left(S_{a d v}\right)$ the threshold applied to a population of $N f(1+\alpha)$ for advanced nodes. The average number of $\mathrm{CHs}$ for intermediate nodes per round per epoch is

$$
N f P_{a d v}
$$

Here, $f$ is the proportion of advanced nodes to the total number of nodes $N$ and $P_{a d v}$ is the weight probability for advanced node.

\section{Performance Evaluation}

To evaluate the performance, we simulated the LEACH Protocol, ILEACH Protocol under two-level homogeneity, and CogILEACH Protocol by MATLAB.

\subsection{Simulation Parameters}

The basic simulation parameters used are listed in Table 1. 
Table 1: Simulation Parameter

\begin{tabular}{|c|c|}
\hline Parameter & Value \\
\hline Cognitive Radio Sensor Networks & $100 \times 100$ \\
\hline Energy consumption by the amplifier to transmit at a shorter distance $\left(\varepsilon_{f s}\right)$ & $10 \mathrm{pJ} / \mathrm{bit} / \mathrm{m} 2$ \\
\hline Energy consumption by the amplifier to transmit at a longer distance $\left(\varepsilon_{\text {amp }}\right)$ & $0.0013 \mathrm{pJ} / \mathrm{bit} / \mathrm{m} 4$ \\
\hline Energy consumed in the electronics circuit to the transmitter or receiver of the signal $\left(E_{\text {elec }}\right)$ & $50 \mathrm{n} / / \mathrm{bit}$ \\
\hline Initial energy of Cognitive sensor node $\left(E_{0}\right)$ & $0.25 \mathrm{~J}, 0.50 \mathrm{~J}$ \\
\hline CRSN node number & 100 \\
\hline Data package size & 500 bytes \\
\hline Packet header & 25 bytes \\
\hline Position of sink & $(50,50)$ \\
\hline Transmitter/receiver antenna height & $0.082 \mathrm{~m}$ \\
\hline
\end{tabular}

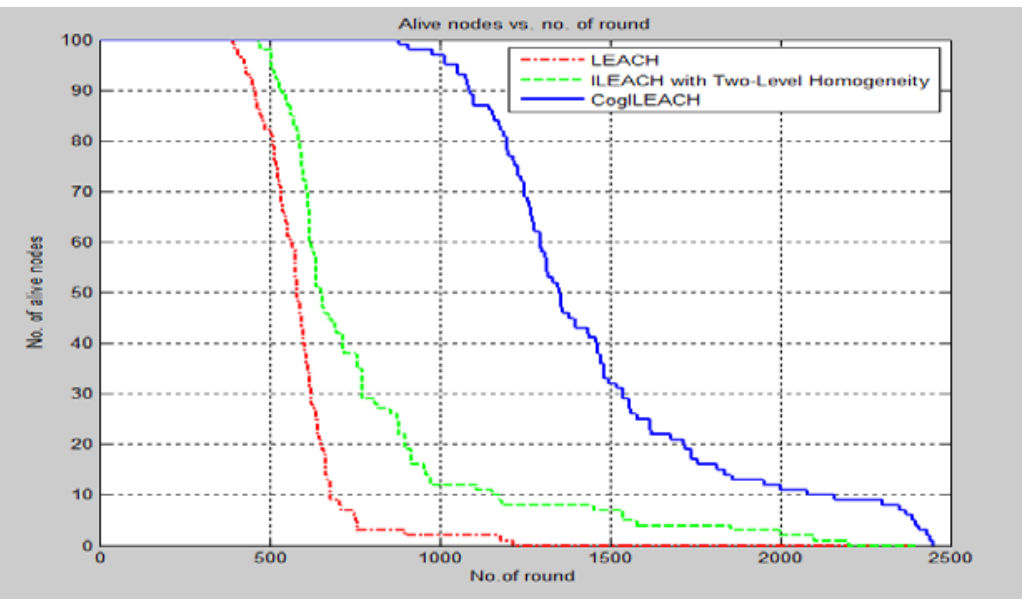

Figure 2: The number of alive nodes versus the number of rounds with $0.25 \mathrm{~J}$

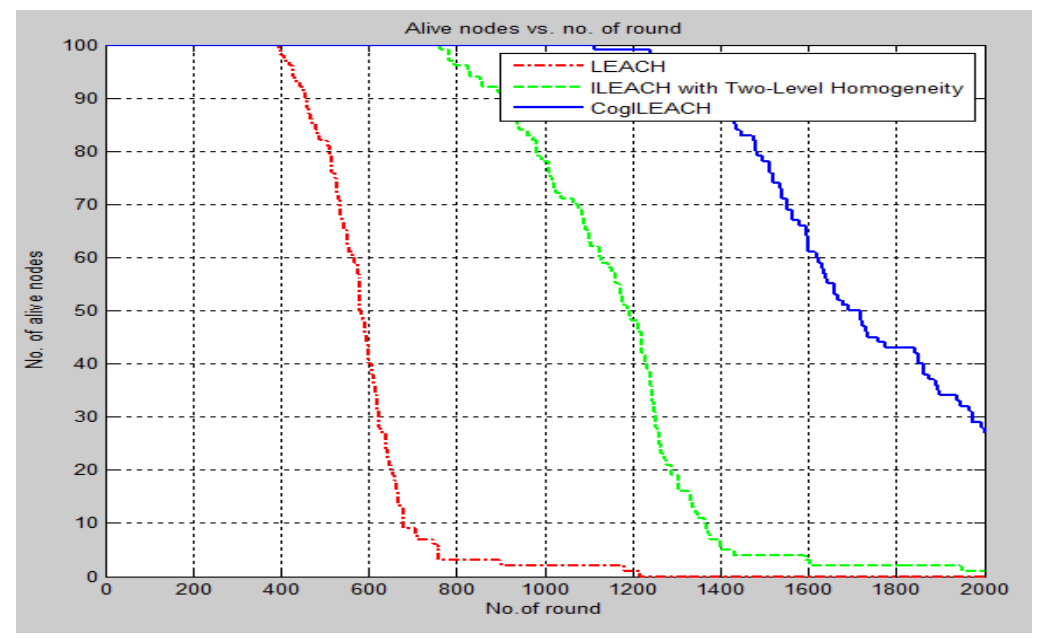

Figure 3: The number of alive nodes versus the number of rounds with $0.5 \mathrm{~J}$

\section{Results Analysis and Discussion}

Figure 1.2 shows the number of alive nodes per round for nodes running CogILEACH, ILEACH under twolevel homogeneity and LEACH protocol. Nodes running CogILEACH protocol lasts longer than those running $\mathrm{LEACH}$ protocol and ILEACH under two-level homogeneity. Figure shows that when the 
Md Sipon Miah, Dilruba Sharmin and Md Mahbubur Rahman; Cognitive Improved LEACH (CogILEACH) Protocol for Wireless Sensor Network, Transactions on Networks and Communications, Volume 4 No. 6, December (2016); pp: 1-9

networking size was $100 \times 100,100$ nodes with initial energy of $0.25 \mathrm{~J}$, the first dead node appeared at round 400 in $\mathrm{LEACH}$, the first dead node appeared at round 480 in ILEACH under two-level homogeneity, and the first dead node appears after round 820 in CogILEACH protocol. Therefore, the performance of the propose CogILEACH protocol is better than the LEACH and the ILEACH protocol under two-level homogeneity.

Figure1.3 shows that when the networking size is $100 \times 100,100$ nodes with initial energy of $0.5 \mathrm{~J}$, the first dead node appears at round 400 in LEACH, the first dead node appears after round 760 in ILEACH under two-level homogeneity, and the first dead node appears after round 1100 in CogILEACH protocol. Therefore, the performance of the propose CogILEACH protocol is better than the LEACH and the ILEACH protocol under two-level homogeneity.

\section{Conclusion}

The proposed CogILEACH protocol shows a significant improvement in the network life time than that of either the conventional LEACH protocol scheme or the ILEACH protocol under two-level homogeneity scheme.

\section{REFERENCES}

[1] Cavalcanti, D., et al., Cognitive radio based wireless sensor networks. Computer Communication and Networks, IEEE International Conference on, 2008. p. 1-6.

[2] Akan, B., et al., Cognitive radio sensor networks. IEEE Netw. 23(4), 34-40 (2009).

[3] KLA Yau, P Komisarczuk, PD Teal, in IEEE 34th Conference on Local Computer Networks. Cognitive radio-based wireless sensor networks: conceptual design and open issues, (2009), pp. 955-962.

[4] Cluster based Energy Efficient Sensing for Cognitive Radio Sensor Networks Usman Mansoor, Department of Telecom Engineering ICT Islamabad affiliated with UET Peshawar Muhammad Khalil Shahid Associate Professor ICT PTCL Academy Islamabad International Journal of Computer Applications (0975 - 8887) Volume 88 - No.7, February 2014.

[5] Abolarinwa J.A, SalawuN and Achonu A, Cognitive Radio-based Wireless Sensor As Next Generation Sensor Network: Concept, Problems and Prospects, Journal of Emerging Trends in Computing and Information Sciences, ISSN 2079-8407, Vol.4, No.8 August 2013.

[6] Rauniyar, Ashish, Jae Min Jang, and Soo Young Shin. "Optimal Hard Decision Fusion Rule for Centralized and Decentralized Cooperative Spectrum Sensing in Cognitive Radio Networks." Journal of Advances in Computer Networks 3.3 (2015).

[7] Proceedings of the UGC Sponsored National Conference on Advanced Networking and Applications, 27th March 2015.Special Issue Published in Int. Jnl. Of Advanced Networking and Applications (IJANA) Page 1 A Note On: Cognitive Radio and It Performance Tools Ms.L.Indhumathi1 M.Phil-Research Scholar, Department of Information Technology, Bharathiar University.

[8] http://searchnetworking.techtarget.com/definition/cognitive-radio. 
[9] Analysis of Spectrum Sensing By Using Energy Detection Technique in Cognitive Radio Kirtibala Shinde1, Madhukar Deshmukh2 1Department of E\&TC, SavitribaiPhule Pune University, Pune, 2Department of E\&TC, SavitribaiPhule Pune University, Pune International Journal of Engineering Inventions e-ISSN: 2278-7461, p-ISSN: 2319-6491 , Volume 4, Issue 8 [January 2015] PP: 33-40

[10] An Energy-Efficient and Compact Clustering Scheme with Temporary Support Nodes For Cognitive RadioSensor Networks Shelly Salim, Sangman Moh *, Dongmin Choi And Ilyong Chung, Department of Computer Engineering, Chosun University, 309 Pilmun- daero, Dong-gu, Gwangju 501-759, Korea

[11] N. M. A. Latiff, C. C. Tsimenidis, and B. S. Sharif, "Performance comparison of Optimization algorithms for clustering in wireless sensor networks," in Proc. of the IEEE International Conference on Mobile Adhoc and Sensor Systems, Pisa, Italy, pp. 1-4, 2007.

[12] Z. Zhang and X. Zhang, "Research of improved clustering routing algorithm based on Load balance in wireless sensor networks," in Proc. Of the IET International Communication Conference on Wireless Mobile and Computing, Shanghai, China, pp. 661-664, 2009.

[13] M. Tong, "LEACH-B: An improved LEACH protocol for wireless sensor network," in Proc.6th International Conference on Wireless Communications Networking and Mobile Computing (WiCOM), Shanghai, China, 2010.

[14] A Survey on LEACH-Based Energy Aware Protocols for Wireless Sensor Networks Raed M. Bani Hani1 and Abdalraheem A. Ijjeh2 1Jordan University of Science and Technology/Network Engineering and Security, Irbid, Jordan 2Jordan University of Science and Technology/Computer Engineering, Irbid, Jordan Journal of Communications Vol. 8, No.3, March 2013.

[15] A. Beni Hssane, M. L. Hasnaoui, M. Saadi, S. Benkirane, and M. Laghdir "Equitable LEACH-E protocol for heterogeneous wireless sensor networks," Intelligent Distributed Computing IV, Studies in Computational Intelligence, vol. 315, pp 171-176, 2010.

[16] L. Qing, Q. Zhu, and M. Wang, "Design of a distributed energy-efficient Clustering algorithm for heterogeneous wireless sensor networks," Computer Communications, vol. 29, no. 12, pp. 2230-2237, 2006.

[17] S. Lindsey and C. S. Raghavendra, "PEGASIS: power-efficient gathering in sensor information systems," In Proc. of IEEE Aerospace Conference, Big Sky: MT, pp. 1125-1130, 2002.

[18] S. Younis and S. Fahmy, "Distributed clustering in ad-hoc sensor networks," in Proc. of IEEE INFOCOM, March, 2004.

[19] G. Smaragdakis, I. Matta, and A. Bestavros, "SEP: a stable election Protocol clustered heterogeneous wireless sensor networks," in Proc. of the International Workshop on Sensor and Actor Network Protocols and Applications (SANPA), 2004.

[20] LEAUCH: low-energy adaptive uneven clustering hierarchy for cognitive radio sensor network Errong Pei1*, Haozhe Han1, Zehua Sun1, Bin Shen1 and Tianqi Zhang2 Pei et al.EURASIP Journal on Wireless Communications and Networking (2015) 2015:122

[21] M. Bradonji and L. Lazos, "Graph-based criteria for spectrum-aware clustering in cognitive radio networks," Ad Hoc Networks, vol. 10, no. 1,pp. 75 - 94, 2012.

[22] H. Zhang, Z. Zhang, H. Dai, R. Yin, and X. Chen, "Distributed spectrum-aware Clustering in cognitive radio sensor networks," in Global Telecommunications Conference (GLOBECOM 2011), 2011 IEEE, pp. 1-6, 2011. 